\title{
Experimental Search for Solar Axions via Coherent Primakoff Conversion in a Germanium Spectrometer
}

\author{
F. T. Avignone III, ${ }^{1}$ D. Abriola, ${ }^{2}$ R. L. Brodzinski, ${ }^{3}$ J. I. Collar, ${ }^{4}$ R. J. Creswick, ${ }^{1}$ D. E. DiGregorio, ${ }^{2}$ H. A. Farach, ${ }^{1}$ \\ A. O. Gattone, ${ }^{2}$ C. K. Guérard, ${ }^{1,2}$ F. Hasenbalg, ${ }^{2}$ H. Huck,${ }^{2}$ H. S. Miley, ${ }^{3}$ A. Morales, ${ }^{5}$ J. Morales, ${ }^{5}$ S. Nussinov, ${ }^{6}$ \\ A. Ortiz de Solórzano, ${ }^{5}$ J. H. Reeves, ${ }^{3}$ J. A. Villar, ${ }^{5}$ and K. Zioutas ${ }^{7}$ \\ (SOLAX Collaboration) \\ ${ }^{1}$ Department of Physics and Astronomy, University of South Carolina, Columbia, South Carolina 29208 \\ ${ }^{2}$ Department of Physics, TANDAR Laboratory, C.N.E.A., Buenos Aires, Argentina \\ ${ }^{3}$ Pacific Northwest National Laboratory, Richland, Washington 99352 \\ ${ }^{4}$ CERN, CH-1211 Geneva, 23 Switzerland \\ ${ }^{5}$ Laboratorio de Física Nuclear y Altas Energias, Universidad de Zaragoza, Zaragoza, Spain \\ ${ }^{6}$ Department of Physics, Tel Aviv University, Tel Aviv, Israel \\ ${ }^{7}$ Department of Physics, University of Thessaloniki, GR54006 Thessaloniki, Greece
}

(Received 17 June 1998)

\begin{abstract}
Results are reported of an experimental search for the unique, rapidly varying temporal pattern of solar axions coherently converting into photons via the Primakoff effect in a single crystal germanium detector when axions are incident at a Bragg angle with a crystalline plane. The analysis of $1.94 \mathrm{~kg} \mathrm{yr}$ of data from the $1 \mathrm{~kg}$ DEMOS detector in Sierra Grande, Argentina, yields a new laboratory bound by an axion-photon coupling of $g_{a \gamma \gamma}<2.7 \times 10^{-9} \mathrm{GeV}^{-1}$, independent of axion mass up to $\sim 1 \mathrm{keV}$. [S0031-9007(98)07812-0]
\end{abstract}

PACS numbers: $14.80 . \mathrm{Mz}, 13.40 .-\mathrm{f}, 96.60 . \mathrm{Vg}$

Early QCD theories predicted a particle with the quantum numbers of the $\eta$ meson, but with a mass close to that of the pion. A term added to the QCD Lagrangian to ameliorate this so-called $\mathrm{U}(1)$ problem violated $C P$ invariance in strong interactions and implied a neutron electric-dipole moment about $10^{9}$ times larger than the experimental upper bound [1]. Peccei and Quinn [2] introduced a new field causing strong $C P$ violation to vanish dynamically. Subsequently, Weinberg [3,4] and Wilczek [5] demonstrated that the Peccei-Quinn mechanism generates a Nambu-Goldstone boson, the axion, that couples to a two-photon vertex via a coupling $g_{a \gamma \gamma}$. Axion production via the Primakoff effect occurs when a photon couples to a charge via a virtual photon, producing an axion. Detection can occur by observing photons resulting from axions coupling to electrical charges via virtual photons.

The dense volume of photons and charges in the sun or any star produces conditions for axion production. The Ge detector then can act as the axion-photon converter and detector. When the characteristic wavelength of the axion satisfies a Bragg condition in the single crystal Ge detector, photon production would occur with an expected temporal pattern depending on the changing relative directions between the vectors from the solar core and the crystalline planes.

Extensive reviews of axion phenomenology and their effects on stellar evolution have been given by Raffelt $[6,7]$ who gives a bound of $10^{-10} \mathrm{GeV}^{-1}$ on the coupling of axions to the two-photon vertex from the population of red giant stars. A detailed treatment of solar axions and of a proposed method of detecting them was given by van Bibber et al. [8]. Details of a theory for searching for axions with germanium detectors were recently given by Creswick et al. [9]. The objective is to detect solar axions through their coherent Primakoff conversion into photons in the lattice of a germanium crystal when the incident angle satisfies the Bragg condition. The detection rates in various energy windows are correlated with the relative orientations of the detector and the sun [9]. This correlation results in a distinctive, unique signature of the axion. In this Letter, the results of a search using a $1 \mathrm{~kg}$, ultralow background germanium detector installed in the HIPARSA iron mine in Sierra Grande, Argentina, at $41^{\circ}$ $41^{\prime} 24^{\prime \prime} \mathrm{S}$ and $65^{\circ} 22^{\prime} \mathrm{W}$ are presented. A description of the experimental setup and detector spectrum was given earlier by Di Gregorio et al. [10] and by Abriola et al. [11]. This experiment was motivated by papers by Buchmüller and Hoogeveen [12] and by Paschos and Zioutas [13]; the present technique was suggested by Zioutas and developed by Creswick et al. [9]. The original technique for searching for solar axions with magnetic helioscopes was presented by Sikivie [14].

Single Ge crystals are grown with an axis of symmetry along the (100) axis, but this detector was constructed originally for another purpose, so the (010) and (001) axes relative to the cryostat were not determined before assembly, as they should be in future experiments.

Therefore, to place a bound on the axion interaction rate, the data were analyzed for many azimuthal orientations of the crystal, and the weakest bound was selected. 
The terrestrial energy spectrum and flux of axions from the sun can be approximated by the expression $[8,9]$ :

$$
\frac{d \Phi}{d E}=\lambda^{1 / 2} \Phi_{0} \frac{\left(E / E_{0}\right)^{3}}{E_{0}\left(e^{E / E_{0}}-1\right)},
$$

where $\lambda=\left(g_{a \gamma \gamma} \times 10^{8}\right)^{4}$ and is dimensionless, $E_{0}=$ $1.103 \mathrm{keV}$, and $\Phi_{0}=5.95 \times 10^{14} \mathrm{~cm}^{-2} \mathrm{sec}^{-1}$. The total flux for $\lambda=1$ integrated from 0 to $12 \mathrm{keV}$ is $3.54 \times$ $10^{15} \mathrm{~cm}^{-2} \mathrm{sec}^{-1}$. The spectrum is a continuum peaking at about $4 \mathrm{keV}$ decreasing to a negligible contribution above $8 \mathrm{keV}$. The differential cross section for Primakoff conversion on an atom with nuclear charge $Z e$ is [9]

$$
\frac{d \sigma}{d \Omega}=\left[\frac{Z^{2} \alpha \hbar^{2} c^{2} g_{a \gamma \gamma}^{2}}{16 \pi}\right] \frac{q^{2}\left(4 k^{2}-q^{2}\right)}{\left(q^{2}+r_{0}^{-2}\right)^{2}},
$$

where $q$ is the momentum transfer, $k$ is the momentum of the incoming axion, and $r_{0}$ is the screening length of the atom in the lattice. For germanium, $\sigma_{0}=Z^{2} \alpha \hbar^{2} c^{2} g_{a \gamma \gamma}^{2} /$ $8 \pi=1.15 \times 10^{-44} \mathrm{~cm}^{2}$ when $g_{a \gamma \gamma}=10^{-8} \mathrm{GeV}^{-1}$, or equivalently $\lambda=1$.

For light axions, the Primakoff process in a periodic lattice is coherent when the Bragg condition $(2 d \sin \theta=$ $n \lambda$ ) is satisfied, or when $\vec{q}$ transferred to the crystal is a reciprocal lattice vector $\vec{G}=2 \pi(h, k, l) / a_{0}$. Here $a_{0}$ is the size of the conventional cubic cell, and $h, k$, and $l$ are integers [15].

It was shown that the rate of conversion of axions with energy $E$ when the sun is in the direction $\hat{k}, \dot{N}(\hat{k}, E)$, can be written [9]

$$
\begin{aligned}
\dot{N}(\hat{k}, E)= & 2 \hbar c \frac{V}{v_{c}^{2}} \sum_{G}|S(G)|^{2} \frac{d \sigma}{d \Omega}(\vec{G}) \frac{1}{|\vec{G}|^{2}} \frac{d \Phi}{d E} \delta \\
& \times\left(E-\frac{\hbar c|\vec{G}|^{2}}{2 \hat{k} \cdot \vec{G}}\right),
\end{aligned}
$$

where $V$ is the volume of the crystal, $v_{c}$ is the volume of a unit cell, $S(G)$ is the structure function for germanium, and $d \Phi / d E$ is evaluated at the axion energy of $\hbar c|\vec{G}|^{2} / 2 \hat{k} \cdot \vec{G}$. The structure function for germanium is

$$
\begin{aligned}
S(G)= & {\left[1+e^{i \pi(h+k+l) / 2}\right] } \\
& \times\left[1+e^{i \pi(h+k)}+e^{i \pi(h+l)}+e^{i \pi(k+l)}\right] .
\end{aligned}
$$

Note that in (3) the coherent conversion of axions occurs only for a particular axion energy given the position of the sun $\hat{k}$ and reciprocal lattice vector $\vec{G}$. However, the detector has a finite energy resolution; for the detector in Sierra Grande it is $1 \mathrm{keV}$ FWHM at $10 \mathrm{keV}$. We take this into account by smoothing $\dot{N}(\hat{k}, E)$ with a Gaussian of the appropriate width. Finally, we take the relevant part of the energy spectrum, in this case from the threshold energy of 4 up to $8 \mathrm{keV}$ (which is just below the $\mathrm{x}$ rays at $10 \mathrm{keV}$ ), and calculate the total rate of conversion in windows of width $\Delta E$, typically $0.5 \mathrm{keV}$,

$$
\begin{aligned}
R(\hat{k}, E)=2 \hbar c & \frac{V}{v_{c}} \sum_{G}|S(G)|^{2} \frac{d \sigma}{d \Omega} \frac{1}{|\vec{G}|^{2}} \frac{d \Phi}{d E} \frac{1}{2} \\
\times & {\left[\operatorname{erf}\left(\frac{E-E_{a}(\hat{k}, \vec{G})}{\sqrt{2} \sigma}\right)\right.} \\
& \left.-\operatorname{erf}\left(\frac{E-E_{a}(\hat{k}, \vec{G})-\Delta E}{\sqrt{2} \sigma}\right)\right],
\end{aligned}
$$

where $E_{a}(\hat{k}, G)=\hbar c|G|^{2} / 2 \hat{k} \cdot \vec{G}$ and $\operatorname{erf}(x)=2 / \sqrt{\pi} \times$ $\int e^{-t^{2}} d t$ is the error function. In Eq. (5) we have neglected the angular size of the core of the sun and the mass of the axion which is justified when $m_{a} c^{2}$ is small compared to the core temperature of the sun [12], i.e., up to a few keV. In Eq. (5), $\sigma$ is the width of the error function.

The theoretical axion detection rate for this detector, calculated with Eq. (5), is shown in Fig. 1. The position of the sun is computed at any instant in time using the U.S. Naval Observatory Subroutines (NOVAS) [16]. The pronounced variation in $R(\hat{k}, E)$ as a function of time invites the data to be analyzed with the correlation function:

$$
\chi \equiv \sum_{i=1}^{n}\left[R\left(t_{i}, E\right)-\langle R(E)\rangle\right] n\left(t_{i}\right)
$$

where $R\left(t_{i}, E\right)$ is the smooth shape of the theoretical rate at the instant of time, $t_{i},\langle R(E)\rangle$ is the average rate over a finite time interval, and $n\left(t_{i}\right)$ is the number of events at $t_{i}$ in a time interval $\Delta t$, usually 0 or 1 . The choice for the weighting function $W(t, E)=R[\hat{k}(t), E]-\langle R(E)\rangle$ is motivated by the requirement that any constant background average to zero in $\chi$, whereas a counting rate which follows $R[\hat{k}(t), E]$ increases $\chi$.

The number of counts at time $t$ in the interval $\Delta t$ is assumed to be due in part to axions and in part to background governed by a Poisson process with mean,

$$
\langle n(t)\rangle=[\lambda R(t, E)+b(E)] \Delta t,
$$

where $b(E)$ is constant in time.

The average value of $\chi$ is then

$$
\langle\chi\rangle=\sum_{i} W\left(t_{i}, E\right)\left[\lambda R\left(t_{i}, E\right)+b(E)\right] \Delta t .
$$

We can add and subtract the constant quantity $\lambda\langle R(E)\rangle$ to the second factor in Eq. (8). Any time independent contributions multiplied by $W(t, E)$ in Eq. (8) and summed over time will vanish. Accordingly, taking the limit as $\Delta t \rightarrow 0$, we obtain

$$
\langle\chi(\lambda)\rangle=\lambda \int_{0}^{T} W^{2}(t, E) d t .
$$



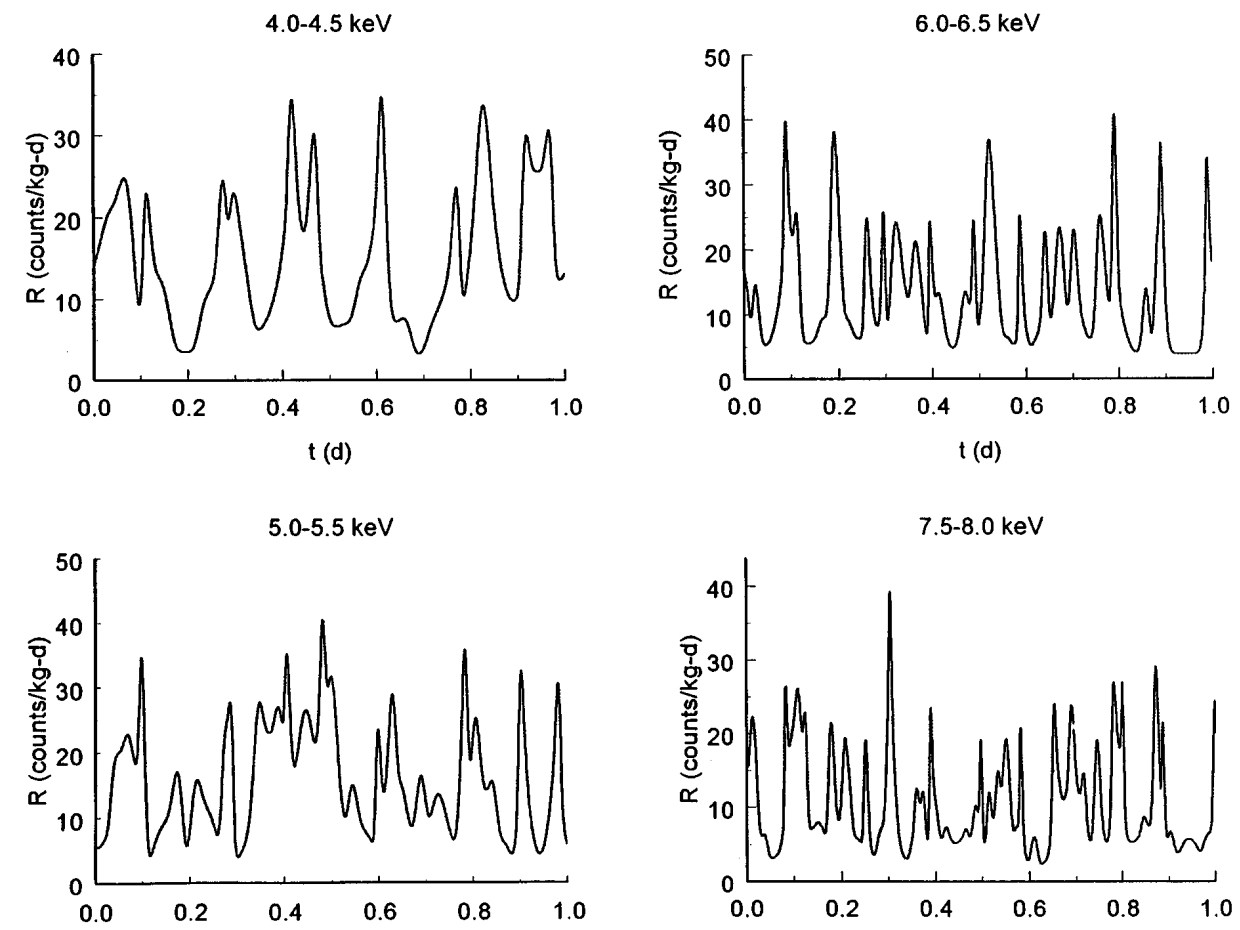

FIG. 1. A typical axion-photon conversion rate $R(t, E)$ for various energy bands. The experimental energy resolution $\mathrm{FWHM}=1.0 \mathrm{keV}$ at $10 \mathrm{keV}$ was used. The time scale is from 0.0 to 1.0 days for each graph. Here $R(t, E)$ was calculated for $g_{a \gamma \gamma}=10^{-8} \mathrm{GeV}^{-1}$.

The expected uncertainty in $\chi,(\Delta \chi)^{2}=\left\langle\chi^{2}\right\rangle-\langle\chi\rangle^{2}$, can be shown to be

$$
(\Delta \chi)^{2}=\sum_{i} W^{2}\left(t_{i}, E\right)\left[\left\langle n\left(t_{i}\right)^{2}\right\rangle-\left\langle n\left(t_{i}\right)\right\rangle^{2}\right]
$$

where the square bracket is $\left\langle\Delta n\left(t_{i}\right)\right\rangle^{2}$, which in Poisson statistics is $\left\langle n\left(t_{i}\right)\right\rangle$. Accordingly,

$$
(\Delta \chi)^{2}=\sum_{i} W^{2}\left(t_{i}, E\right)\left\langle n\left(t_{i}\right)\right\rangle .
$$

By (7) we have

$$
\begin{aligned}
(\Delta \chi)^{2}=\sum_{i} W^{2}\left(t_{i}, E\right)\{ & \lambda\left[R\left(t_{i}, E\right)-\langle R(E)\rangle\right] \\
& +\lambda\langle R(E)\rangle+b(E)\} \Delta t,
\end{aligned}
$$

which in the limit $\Delta t \rightarrow 0$ becomes

$$
(\Delta \chi)^{2}=\lambda \int_{0}^{T} W^{3}(t, E) d t+R_{T}(E) \int_{0}^{T} W^{2}(t, E) d t .
$$

The quantity $R_{T}(t, E)$ is the average total counting rate, including both axion conversions and background.

The data are separately analyzed in energy bins, $\Delta E_{k}$, fixed by the detector resolution (FWHM $\sim 1 \mathrm{keV}$ in this case). The likelihood function is then constructed:

$$
L(\lambda)=\prod_{k} \exp \left[\frac{-\left(\chi_{k}-\left\langle\chi_{k}\right\rangle\right)^{2}}{2\left(\Delta \chi_{k}\right)^{2}}\right] .
$$

To an excellent approximation $\left(\Delta \chi_{k}\right)^{2}$ is dominated by the background.

As a test, typical results for the likelihood function for the cases $\lambda=0$ (no axions) and $\lambda=0.003$ were calculated for the DEMOS detector for one year. The correlation function analysis is sensitive to the presence

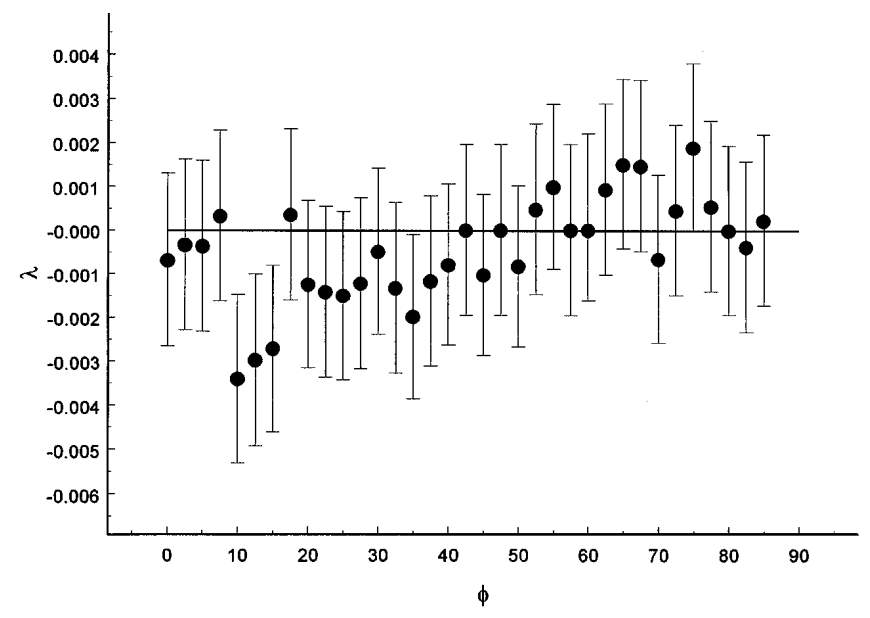

FIG. 2. Values of $\lambda$ calculated from the 707 days of data as a function of the azimuthal angle $\phi$. The error bars are $1 \sigma$. 


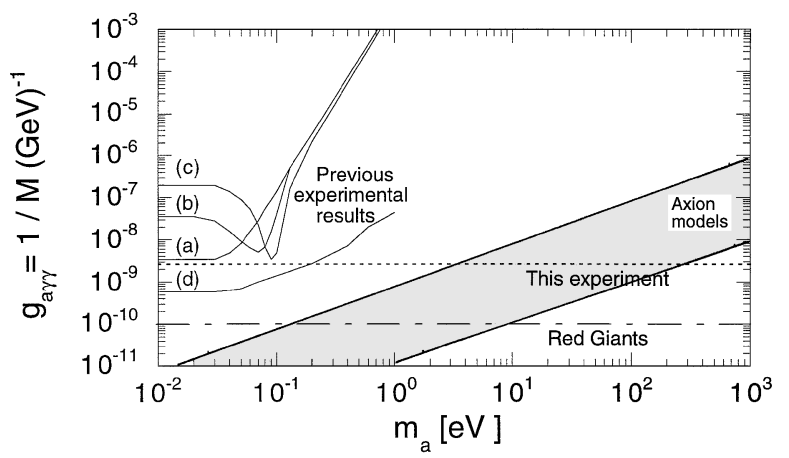

FIG. 3. Exclusion plots on the $g_{a \gamma \gamma}$ vs axion-mass plane. The curves $(a),(b)$, and $(c)$ are from Ref. [17]. Curve $(d)$ is a recent result from Ref. [24].

of a variation in the counting range due to solar axions with a signal to noise ratio less than $1 \%$.

Because the azimuthal orientation of the germanium crystal is not known, the analysis was carried out as a function of the angle $\phi$, the angle between the (010) axis and true north. The results for 707 days of data in the energy range from 4 to 8 in $0.5 \mathrm{keV}$ intervals are shown in Fig. 2.

The most conservative upper bound on $\lambda$, or equivalently $g_{a \gamma \gamma}$, is found by taking for $\phi$ the angle at which $\lambda$ is maximum. This yields an upper bound on the axionphoton coupling constant $g_{a \gamma \gamma}<2.7 \times 10^{-9} \mathrm{GeV}^{-1}$ at the $95 \%$ confidence level.

In Fig. 3 we show the area of the axion-mass-coupling constant plane excluded by this result along with results of earlier work. The curves labeled $(a),(b)$, and $(c)$ are from the magnetic helioscope search by Lazarus et al. [17].

While the present bound is interesting because it is a laboratory constraint, it does not challenge the bound placed by Raffelt [7], $g_{a \gamma \gamma} \leq 10^{-10} \mathrm{GeV}^{-1}$ based on the helium burning rate in low mass stars.

The present technique could also be applied to low temperature sapphire detectors planned for dark matter searches [18] and to giant tellurium oxide arrays [19] and Ge [20] being constructed for double-beta and dark matter searches.

Since the completion of this work, a giant array of Ge detectors GENIUS (Germanium in Nitrogen Underground Setup) was proposed [21] with about $10^{3} \mathrm{~kg}$ of detector mass directly in liquid nitrogen. If their predicted sensitivity for cold dark matter is realistic, we estimate that as an axion experiment it could improve the sensitivity of $g_{a \gamma \gamma}$ significantly.

Recently, E. Fiorini et al. [22] have proposed a highly segmented cryogenic detector of 1020 crystals of $\mathrm{TeO}_{2}$ with a total fiducial mass of $750 \mathrm{~kg}$. This project called CUORE (Cryogenic Underground Observatory for Rare Events) has great promise as the next generation axion experiment. Calculations by Creswick [23] show that $\mathrm{TeO}_{2}$ is more efficient than Ge for axion-to-photon conversion.
This work was supported by the U.S. National Science Foundation (INT930INT1522), the U.S. Department of Energy (DE-AC06-76RLO 1830), the Consejo Nacional de Investigaciones Científicas y Técnicas and Fundacion Antorchas of Argentina, and the Spanish Agency for Science and Technology (AEN96-1657). S. Nussinov would like to acknowledge a BSFG (Israel-U.S.A. Foundation grant). We also thank the personnel of the HIPARSA iron mine for assistance during the installation and J. A. Bangert of the U.S. Naval Observatory for supplying their vector astronomy subroutines.

[1] N. F. Ramsey, Rep. Prog. Phys. 45, 95 (1982); Phys. Rev. D 50, 1218 (1994).

[2] R. D. Peccei and H. Quinn, Phys. Rev. Lett. 38, 1440 (1977); Phys. Rev. D 16, 1791 (1977).

[3] S. Weinberg, Phys. Rev. D 11, 3583 (1975); The Quantum Theory of Fields (Cambridge Press, New York, 1996), Vol. II, p. 243.

[4] S. Weinberg, Phys. Rev. Lett. 40, 223 (1978).

[5] F. Wilczek, Phys. Rev. Lett. 40, 279 (1978).

[6] G. G. Raffelt, Stars as Laboratories for Fundamental Physics (University of Chicago, Chicago, 1996).

[7] G. G. Raffelt, Phys. Rep. 198, 1 (1990).

[8] K. van Bibber, P. M. McIntyre, D. E. Morris, and G. G. Raffelt, Phys. Rev. D 39, 2089 (1989).

[9] R. J. Creswick et al., Phys. Lett. B 427, 235 (1998).

[10] D. E. Di Gregorio et al., Nucl. Phys. B (Proc. Suppl.) 48, 56 (1996).

[11] D. Abriola et al., Astropart. Phys. 6, 63 (1996).

[12] W. Buchmüller and F. Hoogeveen, Phys. Lett. B 237, 278 (1990).

[13] E. A. Paschos and K. Zioutas, Phys. Lett. B 323, 367 (1994).

[14] P. Sikivie, Phys. Rev. Lett. 51, 1415 (1983).

[15] Charles Kittel, in Introduction to Solid State Physics (Wiley, New York, 1986), 6th ed., p. 29.

[16] G. H. Kaplan et al., Astron. J. 97, 1197 (1989).

[17] D. M. Lazarus et al., Phys. Rev. Lett. 69, 2333 (1992).

[18] P. Colling et al., Nucl. Instrum. Methods Phys. Res., Sect. A 354, 408 (1995).

[19] A. Alessandrello et al., Phys. Lett. B 384, 316 (1996); Phys. Rev. Lett. 77, 3319 (1996).

[20] S. W. Nam et al., in Proceedings of the VII International Workshop on Low Temperature Detectors LTD-7, Munich, Germany, 1997, edited by S. Cooper (MPI Physik, Munich, 1998), p. 217.

[21] H. V. Klapdor-Kleingrothaus, J. Helmig, and M. Hirsch, J. Phys. G 24, 483 (1998).

[22] E. Fiorini, in Proceedings of the International Workshop on Non Accelerator New Physics (NANAP-98), Dubna, Russia, 1997, edited by V.A. Bednykov, V. B. Brudanin, and S.G. Kovalenko (Interperiodic Publishing, Russia, 1997), p. 1243.

[23] F. T. Avignone et al., Proceedings of the International Workshop Identification of Dark Matter IDM-98, Sheffield, England, 1998 (World Scientific, Hong Kong, to be published).

[24] Shigetaka Moriyama et al., Phys. Lett. B 434, 147 (1998). 